\title{
Basal ganglia cavernous malformations: case series and systematic review of surgical management and long-term outcomes
}

\author{
Yiping Li, MD, ${ }^{1}$ Anadjeet Khahera, MD, ${ }^{2}$ Jason Kim, MS, ${ }^{3}$ Mauricio Mandel, MD, PhD, ${ }^{1}$ \\ Summer S. Han, MA, PhD, ${ }^{1,4}$ and Gary K. Steinberg, MD, PhD ${ }^{1}$ \\ 'Department of Neurosurgery and Stanford Stroke Center, Stanford University School of Medicine and Stanford Health Center, \\ Stanford; 'University of California, Irvine, California; ${ }^{3}$ University of Wisconsin School of Medicine, Madison, Wisconsin; and \\ ${ }^{4}$ Department of Medicine, Stanford Center for Biomedical Informatics Research, Stanford University School of Medicine, \\ Stanford, California
}

OBJECTIVE Reports on basal ganglia cavernous malformations (BGCMs) are rare. Here, the authors report on their experience in resecting these malformations to offer insight into this infrequent disease subtype.

METHODS The authors retrospectively reviewed a prospectively managed departmental database of all deep-seated cerebral cavernous malformations (CCMs) treated at Stanford between 1987 and 2019 and included for further analysis those with a radiographic diagnosis of BGCM. Moreover, a systematic literature review was undertaken using the PubMed and Web of Science databases.

RESULTS The departmental database search yielded 331 patients with deep-seated CCMs, 44 of whom had a BGCM (13.3\%). Headache was the most common presenting sign (53.5\%), followed by seizure (32.6\%) and hemiparesis (27.9\%). Lesion location involved the caudate nucleus in $21.4 \%$ of cases compared to $78.6 \%$ of cases within the lentiform nucleus. Caudate BGCMs were larger on presentation and were more likely to present to the ependymal surface $(p<$ 0.001 ) with intraventricular hemorrhage and hydrocephalus ( $p=0.005$ and 0.007 , respectively). Dizziness and diplopia were also more common with lesions involving the caudate. Because of their anatomical location, caudate BGCMs were preferentially treated via an interhemispheric approach and were less likely to be associated with worsening perioperative deficits than lentiform BGCMs ( $p=0.006$ and 0.045 , respectively). Ten patients ( $25.6 \%)$ were clinically worse in the immediate postoperative period, 4 (10.2\%) of whom continued to suffer permanent morbidity at the last follow-up. A longterm good outcome (modified Rankin Scale [mRS] score $0-1$ ) was attained in $74.4 \%$ of cases compared to the $69.2 \%$ of patients who had presented with an mRS score $0-1$. Relative to their presenting mRS score, $89.8 \%$ of patients had an improved or unchanged status at the last follow-up. The median postoperative follow-up was 11 months (range 1-252 months). Patient outcomes after resection did not differ among surgical approaches; however, patients presenting with hemiparesis and lesions involving the globus pallidus or posterior limb of the internal capsule were more likely to suffer neurological deficits during the immediate perioperative period. Patients who had undergone awake surgeries were more likely to suffer neurological decline at the early as well as the late follow-up. When adjusting for awake craniotomy as a potential confounder of lesion location, a BGCM involving the posterior limb was predictive of developing early postoperative deficits, but this finding did not persist at the long-term follow-up.

CONCLUSIONS Surgery is a safe and effective treatment modality for managing BGCMs, with an estimated long-term permanent morbidity rate of around $10 \%$.

https://thejns.org/doi/abs/10.3171/2020.7.JNS2098

KEYWORDS cavernoma; cavernous malformation; basal ganglia; interventional neurosurgery; vascular disorders

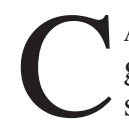

AVERNOUS malformations (CMs) of the basal ganglia are rare, deep-seated lesions that often cause significant neurological deficits (NDs) because of their proximity to eloquent neurovascular structure. $.^{1-5} \mathrm{Re}-$ ports on basal ganglia cavernous malformations (BGCMs) often group these lesions with other deep-seated lesions such as insular, brainstem, and thalamic cavernomas because of the rarity of BGCMs; therefore, few studies have performed a specific analysis of this subset. ${ }^{1,5,6}$ BGCMs present with unique signs and symptoms such as hydro-

ABBREVIATIONS BGCM = basal ganglia CM; CM = cavernous malformation; MEP/SSEP = motor evoked potential/somatosensory evoked potential; mRS = modified Rankin Scale; ND = neurological deficit. 
cephalus and parkinsonism, requiring intricate management decisions, mastery of the adjacent anatomy, and complex surgical approaches. Consequently, BGCMs, like thalamic or brainstem cavernomas, should be separately analyzed to better understand the specific details and considerations for management. In this report, we examine our experience with BGCM and describe anatomical considerations, microsurgical techniques, associated results, and a review of the literature.

\section{Methods \\ Data Collection}

We retrospectively reviewed a prospectively managed departmental database of all deep-seated cerebral CMs treated by the senior author between 1987 and 2019 to find those patients who had a BGCM. We then reviewed the imaging studies of each patient and included only those patients with a confirmed diagnosis of a BGCM, defined as a CM in the caudate nucleus, putamen, or globus pallidus. This search yielded 331 patients with deep-seated lesions, 44 of whom had a BGCM (13.3\%). We reviewed the records of all 44 patients including presenting signs, treatment modality, early surgical results, and long-term outcomes.

\section{Patient Outcomes}

The primary outcome measure was the modified Rankin Scale (mRS) score at the early follow-up (discharge to the 2-week wound check) and the last clinic follow-up. The mRS score was assigned by a physician or nurse clinician. The mRS scores at the early and late follow-ups were compared with presurgical mRS scores. The secondary outcome measure was differences in presenting signs, treatment modality, early surgical results, and long-term outcomes based on lesion location (caudate vs lentiform nucleus).

\section{Statistical Analysis}

Variables included in the analysis were age, sex, lesion size, lesion location, headache, dizziness, memory disturbances, impaired gait, weakness, sensory abnormalities, aphasia, seizures, acute intraparenchymal hemorrhage, intraventricular hemorrhage, hydrocephalus, prior surgery or radiotherapy, surgical approach, and pre- and postsurgical mRS scores. For categorical variables, Fisher's exact test and the chi-square test were used, while a Mann-Whitney U-test was utilized to evaluate continuous variables. Univariate logistic analysis was performed to determine predictors of early and long-term surgical outcomes. The significance for variable removal and entry was set to 0.10 and 0.05 , respectively, and entered into a backward stepwise multivariate logistic regression for two outcome variables: $\mathrm{mRS}$ at the early follow-up (discharge to 2 weeks) and at the last follow-up. The following variables were considered in the stepwise selection process: awake surgery, globus pallidus location, internal capsule location, presence of weakness upon presentation for early follow-up outcomes, and the addition of a decline in the mRS score at the early follow-up for long-term outcomes. Interactions were tested between outcomes and each significant factor. Multiple comparison adjustment was performed using the Bonferroni method to reduce the family-wise error rate. The Hosmer-Lemeshow test was used to check for interactions between outcomes and each significant factor. The likelihood ratio test was used to compare the full model including all the interactions with the reduced model without interactions. Because of the potential confounding effects of awake surgery performed for locations involving eloquent tissue, repeat stepwise selection was applied and the awake surgery variable was removed. Regression analysis was performed using SAS analytics software (SAS Institute) and STATA/SE 15.1 for Windows (StataCorp). Statistical significance was defined as a $\mathrm{p}$ value $<0.05$.

\section{Literature Search}

A systematic review was conducted according to the PRISMA criteria (Fig. 1). Articles were selected by searching the PubMed and Web of Science electronic databases for articles published from 1970 to 2019. Only articles written in the English language were reviewed. Additional review was performed by examining the reference lists of all included articles. The following algorithm was used: ("Basal Ganglia" OR "Caudate" OR "Lentiform" OR "Deep") AND ("Cavernous Malformation" OR "Cavernoma" OR "Cavernous Angioma" OR "Cavernous Hemangioma") AND ("Surgical Outcomes" OR "Surgery"). Given the rarity of BGCM, we included case reports in our analysis, for a total of 31 studies.

\section{Results}

\section{Patient Characteristics}

Between 1987 and 2019, the senior author surgically treated 44 patients with BGCM, 24 men and 20 women (Table 1). The mean patient age was 38 years. Headache was the most common presenting sign (53.5\%), followed by seizure (32.6\%) and hemiparesis (27.9\%). Overall, 38.1\% of the lesions occurred in the dominant hemisphere, with the most common location in the putamen (61.9\%) and a median size of $2 \mathrm{~cm}$. Radiographic evidence of intraparenchymal hemorrhage was identified in $86 \%$ of cases, while intraventricular hemorrhage was identified in $11.6 \%$, with $7.0 \%$ of patients presenting with acute hydrocephalus. No patient required long-term ventricular drainage at the last follow-up.

\section{Lesion Location}

In 9 cases (21.4\%), the lesion was centered within the caudate nucleus compared to $33(78.6 \%)$ within the lentiform nucleus (Table 2). Lesions within the caudate nucleus were more likely to be larger on presentation $(\mathrm{p}=0.039)$, present to the ependymal surface $(\mathrm{p}<0.001)$, and present with intraventricular hemorrhage $(\mathrm{p}=0.005)$ and hydrocephalus $(p=0.007)$. Patients with these lesions were also more likely to present with dizziness $(\mathrm{p}=0.042)$ and diplopia $(p=0.007)$. Compared to lesions within the lentiform nucleus, BGCMs within the caudate were preferentially treated using an interhemispheric approach $(p=0.006)$, and patients with these lesions were less likely to suffer worsening perioperative deficits $(p=0.045)$. Although 


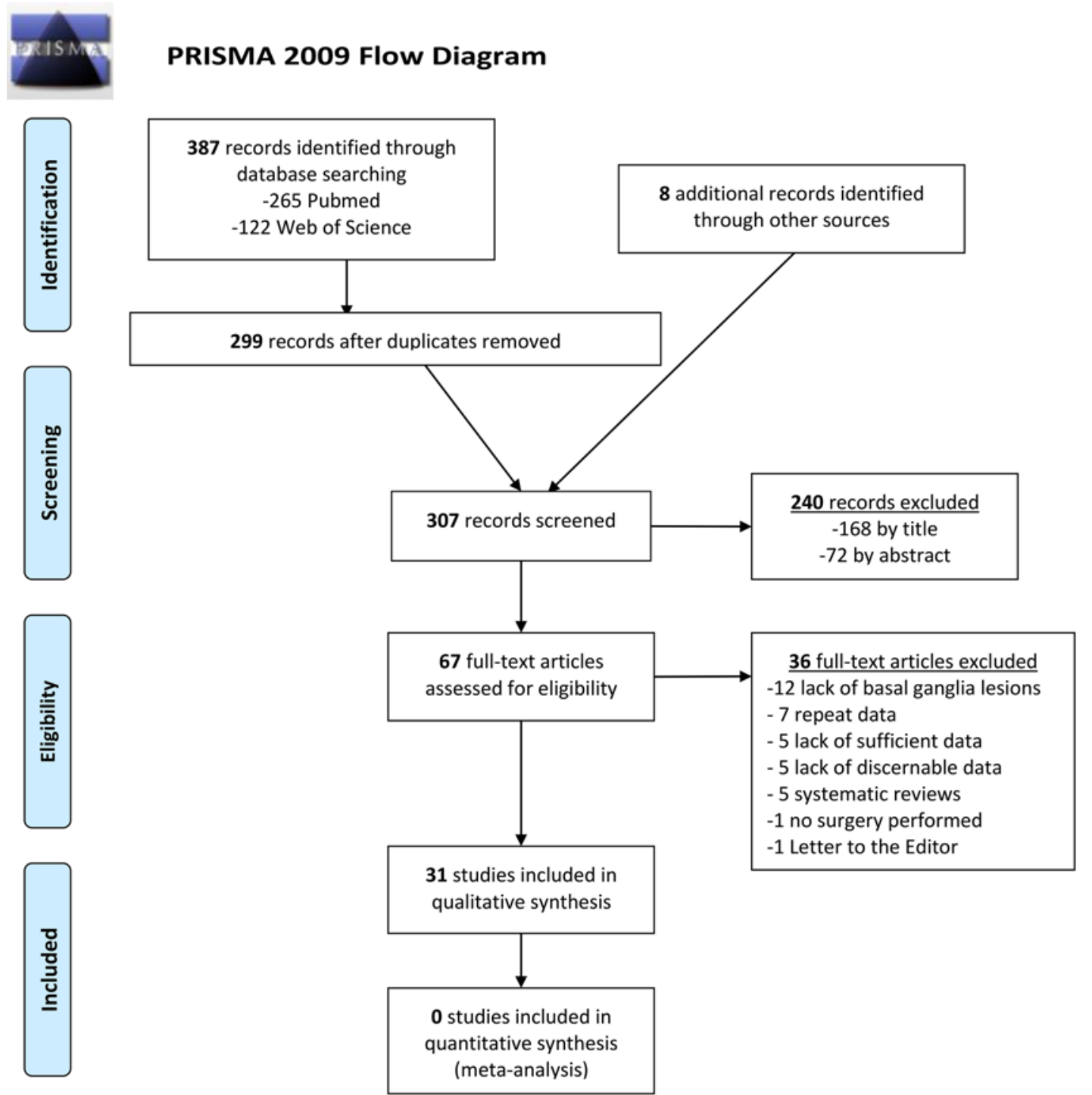

From: Moher D, Liberati A, Tetzlaff J, Altman DG, The PRISMA Group (2009). Preferred Reporting /tems for Systematic Reviews and MetaAnalyses: The PRISMA Statement. PLoS Med 6(7): e1000097. doi:10.1371/journal.pmed1000097

For more information, visit www.prisma-statement.org.

FIG. 1. PRISMA flow diagram.

statistically significant based on a nominal significance threshold of $\alpha=0.05$, this association was not statistically significant after adjusting for multiple comparisons based on the Bonferroni method $(\alpha=0.001)$. Only presentation to the ependymal surface remained statistically significant.

\section{Surgical Management}

The indications for surgery included symptomatic intraparenchymal or intraventricular hemorrhage (42 of 43 patients) or seizure presentation (1 without acute or subacute hemorrhage). Three patients had undergone previous radiosurgery, and none had undergone previous resection. The transsylvian transinsular approach $(55.8 \%)$ was most commonly utilized, followed by the transfrontal transcortical approach (23.3\%; Table 3). Frameless stereo- tactic navigation and motor evoked potential/somatosensory evoked potential (MEP/SSEP) neuromonitoring were used in all cases. Awake craniotomy with motor or speech mapping was performed in $16.7 \%$ of cases. Awake craniotomy was selected for lesions close to the internal capsule's posterior limb or for left-sided surgical trajectories requiring manipulation of language areas. We previously described our surgical technique using a flexible omnidirectional carbon dioxide laser, which was utilized in cases after April 2009.7 We prefer to use the carbon dioxide laser, as we have previously shown that this technique reduces thermal damage to surrounding brain parenchyma compared to that with traditional bipolar coagulation.? Gross-total resection was achieved in $97.7 \%$ of cases, with one residual BGCM identified on postoperative imaging. 
TABLE 1. Patient and lesion characteristics

\begin{tabular}{|c|c|}
\hline & Value \\
\hline Mean age in yrs (range) & $38.5(8-69)$ \\
\hline Male sex & $24 / 44$ \\
\hline Mean lesion size in $\mathrm{cm}$ (range) & $2(0.8-5.5)$ \\
\hline \multicolumn{2}{|l|}{ Lesion location } \\
\hline Nondominant hemisphere & $26 / 42$ \\
\hline Caudate & $9 / 42$ \\
\hline Putamen & $26 / 42$ \\
\hline Globus pallidus & $23 / 42$ \\
\hline IC involvement & $13 / 42$ \\
\hline DVA & $12 / 38$ \\
\hline $\begin{array}{l}\text { Presentation to ependyma (1) or subarachnoid } \\
\text { space (2) or both (3) }\end{array}$ & $27,10,1$ \\
\hline Familial history & $4 / 40$ \\
\hline Lesion $>1 \mathrm{~cm}$ & $24 / 43$ \\
\hline Headache & $23 / 43$ \\
\hline Dizziness & $2 / 43$ \\
\hline Somnolence & $0 / 43$ \\
\hline Memory issue & $3 / 43$ \\
\hline Altered mental status & $5 / 43$ \\
\hline Behavior & $0 / 43$ \\
\hline Impaired gait & $4 / 43$ \\
\hline Weakness & $12 / 43$ \\
\hline Seizure & $14 / 43$ \\
\hline Diplopia & $3 / 43$ \\
\hline Sensory: numbness/pain & $8 / 43$ \\
\hline Aphasia & $4 / 43$ \\
\hline Cranial nerve deficit & $4 / 43$ \\
\hline Hydrocephalus & $3 / 43$ \\
\hline $\mathrm{IPH}$ & $37 / 43$ \\
\hline IVH & $5 / 43$ \\
\hline Median no. of hemorrhages (range) & $1(0-4)$ \\
\hline $\begin{array}{l}\text { Median time of surgery from initial hemorrhage } \\
\text { in wks (range) }\end{array}$ & $14(0.5-1144)$ \\
\hline $\begin{array}{l}\text { No previous surgery (1), previous surgery (2), } \\
\text { or previous radiation ( } 3 \text { ) }\end{array}$ & $40,0,3$ \\
\hline Median FU period in mos (range) & $11(1-252)$ \\
\hline
\end{tabular}

DVA = developmental venous anomaly; FU = follow-up; $I C=$ internal capsule; $\mathrm{IPH}=$ intraparenchymal hemorrhage; IVH = intraventricular hemorrhage.

There were no cases of recurrence, rehemorrhage, or reoperation on the index lesion. Three remote and unrelated de novo cavernomas were identified on follow-up imaging, all asymptomatic.

\section{Outcomes}

Ten patients $(25.6 \%)$ were clinically worse after surgery in the early perioperative period, 4 (10.2\%) of whom continued to suffer permanent morbidity at the last followup. There were no surgical deaths in this case series. A long-term good outcome (mRS score 0-1) was achieved in $74.4 \%$ of cases overall, as compared to the $69.2 \%$ of
TABLE 2. Demographics and outcomes based on lesion location among 42 patients

\begin{tabular}{|c|c|c|c|}
\hline & $\begin{array}{l}\text { Caudate } \\
\text { Nucleus }\end{array}$ & $\begin{array}{l}\text { Lentiform } \\
\text { Nucleus }\end{array}$ & $\begin{array}{c}p \\
\text { Value }\end{array}$ \\
\hline Mean age in yrs (range) & $41(22-61)$ & $39(8-69)$ & 0.65 \\
\hline Male sex & $6 / 9$ & $17 / 33$ & 0.48 \\
\hline Mean lesion size in $\mathrm{cm}$ (range) & $3(1-5.5)$ & $2(0.8-4)$ & 0.039 \\
\hline Lesion in nondominant side & $6 / 9$ & $20 / 33$ & 0.99 \\
\hline DVA & $5 / 8$ & $7 / 30$ & 0.081 \\
\hline $\begin{array}{l}\text { Presentation to ependyma (1) } \\
\text { or subarachnoid space (2) or } \\
\text { both (3) }\end{array}$ & $0,8,0$ & $27,2,1$ & $<0.001$ \\
\hline Familial history & $0 / 8$ & $4 / 30$ & 0.560 \\
\hline Lesion $>1 \mathrm{~cm}$ & $5 / 9$ & $19 / 33$ & 0.99 \\
\hline Headache & $6 / 9$ & $17 / 33$ & 0.480 \\
\hline Dizziness & $2 / 9$ & $0 / 33$ & 0.042 \\
\hline Somnolence & $0 / 9$ & $0 / 33$ & 0.99 \\
\hline Memory issue & $1 / 9$ & $2 / 33$ & 0.520 \\
\hline Altered mental status & $3 / 9$ & $2 / 33$ & 0.057 \\
\hline Behavior & $0 / 9$ & $0 / 33$ & 0.99 \\
\hline Gait & $2 / 9$ & $2 / 33$ & 0.200 \\
\hline Weakness & $1 / 9$ & $11 / 33$ & 0.250 \\
\hline Seizure & $2 / 9$ & $11 / 33$ & 0.700 \\
\hline Diplopia & $3 / 9$ & $0 / 33$ & 0.007 \\
\hline Sensory: numbness/pain & $0 / 9$ & $8 / 33$ & 0.170 \\
\hline Aphasia & $1 / 9$ & $3 / 33$ & 0.99 \\
\hline Cranial nerve deficit & $2 / 9$ & $2 / 33$ & 0.200 \\
\hline $\mathrm{IPH}$ & $7 / 9$ & $30 / 33$ & 0.290 \\
\hline IVH & $4 / 9$ & $1 / 33$ & 0.005 \\
\hline Hydrocephalus & $3 / 9$ & $0 / 33$ & 0.007 \\
\hline $\begin{array}{l}\text { Median no. of hemorrhages } \\
\text { (range) }\end{array}$ & $1(0-3)$ & $1(0-4)$ & 0.420 \\
\hline $\begin{array}{l}\text { Median time from bleed to } \\
\text { surgery in wks (range) }\end{array}$ & $2.5(1-4)$ & $20(0.5-1144)$ & 0.092 \\
\hline $\begin{array}{l}\text { No previous surgery (1), previous } \\
\text { surgery (2), or previous radiation } \\
\text { (3) }\end{array}$ & $9,0,0$ & $30,0,3$ & 0.350 \\
\hline $\begin{array}{l}\text { Median preop mRS score } \\
\text { (range) }\end{array}$ & $1(1-5)$ & $1(0-5)$ & 0.970 \\
\hline Surgical approach* & $1,3,1,4$ & $22,1,1,9$ & 0.006 \\
\hline Worsening deficit perioperatively & $1 / 8$ & $17 / 30$ & 0.045 \\
\hline Awake surgery & $0 / 9$ & $7 / 33$ & 0.31 \\
\hline Periop hemorrhage or stroke & $0 / 8$ & $4 / 30$ & 0.560 \\
\hline Median FU period in mos (range) & $4(1-91)$ & $16(0-176)$ & 0.140 \\
\hline Residual lesion & $0 / 8$ & $1 / 30$ & 0.99 \\
\hline Recurrence & $0 / 8$ & $0 / 30$ & 0.99 \\
\hline New lesion & $1 / 8$ & $2 / 30$ & 0.520 \\
\hline Reoperation & $0 / 8$ & $1 / 30$ & 0.99 \\
\hline $\begin{array}{l}\text { Median early FU mRS score } \\
\text { (range) }\end{array}$ & $0(0-4)$ & $1(0-5)$ & 0.120 \\
\hline Median last mRS score (range) & $0(0-1)$ & $1(0-5)$ & 0.064 \\
\hline
\end{tabular}

Boldface type indicates statistical significance.

* Transsylvian, transcallosal, supracarotid infrafrontal, transfrontal transcortical. 
TABLE 3. Surgical approach and patient outcomes among 39 patients

\begin{tabular}{ccc}
\hline \multirow{2}{*}{ Approach } & No. of Patients w/ a Worse mRS Score \\
\cline { 2 - 3 } & Early FU & Last FU \\
\hline Transsylvian transinsular & $5 / 24$ & $4 / 24$ \\
\hline Awake & $4 / 24$ & $3 / 24$ \\
\hline Not awake & $1 / 24$ & $1 / 24$ \\
\hline Transcallosal & $0 / 3$ & $0 / 3$ \\
\hline Awake & $0 / 3$ & $0 / 3$ \\
\hline Not awake & $0 / 3$ & $0 / 3$ \\
\hline Contralat transcallosal & - & - \\
\hline Awake & - & - \\
\hline Not awake & - & - \\
\hline Supracarotid infrafrontal & $0 / 2$ & $0 / 2$ \\
\hline Awake & $0 / 2$ & $0 / 2$ \\
\hline Not awake & $0 / 2$ & $0 / 2$ \\
\hline Transfrontal transcortical & $5 / 10$ & $2 / 10$ \\
\hline Awake & $3 / 10$ & $1 / 10$ \\
\hline Not awake & $2 / 10$ & $1 / 10$ \\
\hline
\end{tabular}

patients who had presented with an mRS score $0-1$ (Fig. 2). Relative to their baseline presenting mRS score, $89.8 \%$ of patients had an improved or unchanged status at the last follow-up. The median postoperative follow-up for all patients was 11 months (range 1-252 months). Patient outcomes after resection did not differ among surgical approaches; however, patients presenting with hemiparesis and lesions located within the globus pallidus and involving the posterior limb of the internal capsule were more likely to suffer NDs within the immediate perioperative period, according to univariate analysis (Table 4). On both univariate and multivariate analysis, patients who had undergone awake craniotomy were more likely to suffer from neurological decline at the early as well as the late follow-up (Table 5). When adjusting for awake craniotomy as a potential confounder of lesion location, a BGCM involving the posterior limb was predictive of developing early postoperative deficits (OR 8.56, 95\% CI 1.68-43.5, $\mathrm{p}=0.009$ ), but this finding did not persist at the long-term follow-up. These findings remained statistically significant after adjusting for multiple comparisons $(\alpha=0.01)$.

\section{Systematic Review}

The initial search identified 395 publications satisfying our inclusion criteria. After removing duplicate publications, 307 relevant articles were retrieved and their abstracts screened. Additional review of the references was performed to identify any pertinent articles missed during the initial search process. After title and abstract review, 67 full-text articles were assessed for eligibility. A fulltext review excluded an additional 36 articles, most because of a lack of sufficient data or lesions within the basal ganglia. Extensive review was subsequently performed on the remaining 31 studies for qualitative synthesis (Fig. 1). For results of the systematic review, see Supplementary Tables 1-4.

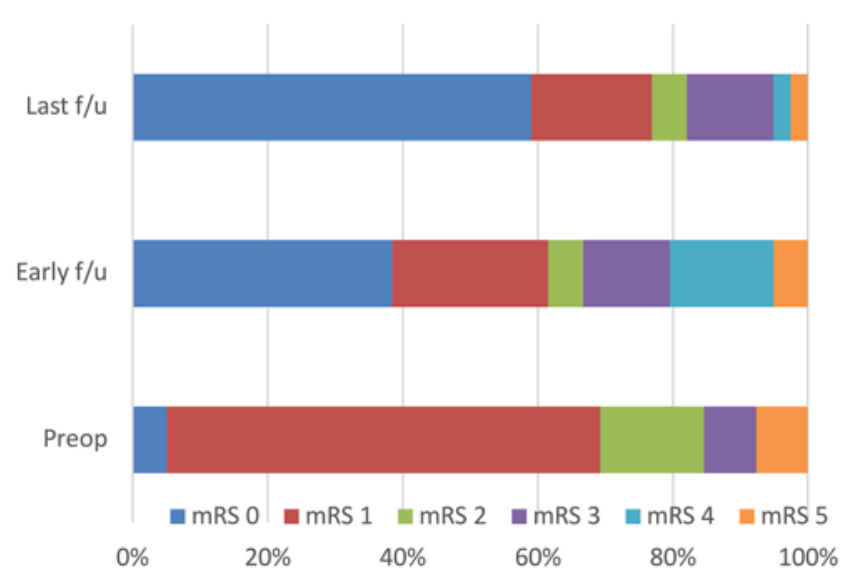

FIG. 2. Neurological outcomes after microsurgical resection of BGCMs. f/u = follow-up.

\section{Illustrative Cases}

The cases described below are from our institutional database.

\section{Case 1}

A 19-year-old male presented to an outside hospital after having a seizure that was witnessed by family. He had no significant prior medical history and was prescribed an antiseizure medication and discharged. A few months later, he had another witnessed seizure. Further workup revealed a $3 \times 2-\mathrm{cm}$ left BGCM (Fig. 3). The patient was found to have mild short-term memory loss, and given the second seizure, he elected to undergo surgical treatment. He underwent a stereotactic left frontal craniotomy via an interhemispheric transcallosal approach for microscopic computer-assisted carbon dioxide laser resection of this CM under mild hypothermia and with intraoperative electrophysiological monitoring. The surgery was uneventful, and the patient was discharged on postoperative day 2 (Video 1).

VIDEO 1. Case example of an interhemispheric approach.

Copyright Yiping Li. Published with permission. Click here to view.

At the 2-week follow-up, the patient was at his neurological baseline. He will begin weaning off his antiseizure medications at 3 months and already notes some improvement in his short-term memory.

\section{Case 2}

A 20-year-old female presented to an outside hospital with syncope. She had had multiple episodes of severe headaches over the few weeks prior to this event. CT scanning performed at an outside facility was concerning for a $3-\mathrm{cm}$, partially thrombosed aneurysm with subacute hemorrhage. Further imaging demonstrated a left BGCM with subacute hemorrhage and a fluid-fluid level (Fig. 4). The patient underwent stereotactic left frontotemporal craniotomy for microscopic computer-assisted resection via a transsylvian transinsular approach and intraoperative electrophysiological functional mapping. The surgery was uneventful, and the patient was discharged on postoperative day 2 (Video 2). 
TABLE 4. Surgical outcomes (mRS score) upon discharge and at the last follow-up among 39 patients

\begin{tabular}{|c|c|c|c|c|c|c|}
\hline \multirow[b]{2}{*}{ Characteristic } & \multicolumn{3}{|c|}{ Early FU } & \multicolumn{3}{|c|}{ Last FU } \\
\hline & Stable/lmproved $(n=29)$ & Decline $(n=10)$ & p Value & Stable/Improved $(n=33)$ & Decline $(n=6)$ & $p$ Value \\
\hline Mean age in yrs (range) & $39(19-69)$ & $40(16-63)$ & 0.747 & $37(16-69)$ & $50.5(20-63)$ & 0.119 \\
\hline Male sex & 19 & 4 & 0.264 & 22 & 5 & 0.428 \\
\hline Mean lesion size in $\mathrm{cm}$ (range) & $2(0.8-5.5)$ & $3(1-4)$ & 0.068 & $2.4(0.8-5.5)$ & $2(1-4)$ & 0.860 \\
\hline \multicolumn{7}{|l|}{ Lesion location } \\
\hline Nondominant side & 18 & 7 & 0.99 & 21 & 4 & 0.99 \\
\hline Caudate & 8 & 0 & 0.082 & 8 & 0 & 0.309 \\
\hline Putamen & 18 & 7 & 0.99 & 21 & 4 & 0.99 \\
\hline Globus pallidus & 11 & 9 & 0.009 & 16 & 4 & 0.663 \\
\hline IC involvement & 6 & 7 & 0.016 & 9 & 4 & 0.154 \\
\hline DVA & 11 & 1 & 0.124 & 11 & 1 & 0.642 \\
\hline Familial history & 2 & 2 & 0.267 & 2 & 2 & 0.104 \\
\hline Headache & 15 & 6 & 0.726 & 17 & 4 & 0.667 \\
\hline Dizziness & 2 & 0 & 0.99 & 2 & 0 & 0.99 \\
\hline Memory issue & 2 & 1 & 0.99 & 2 & 1 & 0.403 \\
\hline Altered mental status & 4 & 1 & 0.99 & 4 & 1 & 0.99 \\
\hline Gait & 3 & 1 & 0.99 & 3 & 1 & 0.502 \\
\hline Weakness & 5 & 6 & 0.017 & 8 & 3 & 0.323 \\
\hline Seizure & 10 & 3 & 0.99 & 11 & 2 & 0.99 \\
\hline Diplopia & 3 & 1 & 0.556 & 3 & 0 & 0.99 \\
\hline Sensory: numbness/pain & 5 & 3 & 0.399 & 7 & 1 & 0.99 \\
\hline Aphasia & 3 & 1 & 0.99 & 3 & 1 & 0.502 \\
\hline Cranial nerve deficit & 2 & 2 & 0.267 & 3 & 1 & 0.502 \\
\hline Hydrocephalus & 2 & 0 & 0.99 & 2 & 0 & 0.99 \\
\hline $\mathrm{IPH}$ & 24 & 9 & 0.99 & 28 & 5 & 0.99 \\
\hline IVH & 4 & 0 & 0.556 & 4 & 0 & 0.99 \\
\hline Mean preop mRS score (range) & $1(0-5)$ & $1.5(0-3)$ & 0.387 & $1(0-5)$ & $1(0-2)$ & 0.203 \\
\hline Mean no. of hemorrhages (range) & $1(0-4)$ & $1(0-3)$ & 0.712 & $1(0-4)$ & $1.5(0-3)$ & 0.372 \\
\hline Mean timing of surgery in wks (range) & $12(0.5-191)$ & $33(4-1144)$ & 0.394 & $16(0.5-191)$ & $14(4-1144)$ & 0.671 \\
\hline Previous surgery or radiation & 3 & 0 & 0.296 & 3 & 0 & 0.448 \\
\hline Awake surgery & 1 & 6 & $<0.001$ & 3 & 4 & 0.006 \\
\hline Surgical approach* & $19,3,2,5$ & $5,0,0,5$ & 0.163 & $20,3,2,8$ & $4,0,0,2$ & 0.776 \\
\hline Mean early FU mRS score (range) & $0(0-5)$ & $3.5(1-5)$ & $<0.001$ & $0(0-5)$ & $3(0-4)$ & 0.140 \\
\hline Residual lesion & 0 & 1 & 0.256 & 0 & 1 & 0.153 \\
\hline Recurrence & 0 & 0 & 0.99 & 0 & 0 & 0.99 \\
\hline Reoperation & 0 & 1 & 0.256 & 0 & 1 & 0.154 \\
\hline Mean FU period in mos (range) & $8(0-252)$ & $19.5(1-176)$ & 0.405 & $11(0-252)$ & $26(2-176)$ & 0.406 \\
\hline
\end{tabular}

Boldface type indicates statistical significance.

* Transsylvian, transcallosal, supracarotid infrafrontal, transfrontal transcortical.

VIDEO 2. Case example of a transsylvian approach. Copyright Yiping Li. Published with permission. Click here to view.

At the long-term follow-up, the patient was at her neurological baseline and is back to school full time.

\section{Discussion}

To the best of our knowledge, this is the largest case series in the literature to analyze the clinical course of surgically managed BGCM. We found that surgery is a safe and effective modality for managing these challeng- ing lesions. Although nearly a quarter of the patients will experience temporary neurological decline in the immediate perioperative period, the permanent morbidity rate was around $10 \%$, with most patients improving or remaining at their neurological baseline by the long-term follow-up. We also noted that patients with lesions involving the posterior limb of the internal capsule were more at risk for developing early perioperative deficits but did not have an increased risk of developing permanent morbidity. Patients with lesions involving the caudate nucleus were also less likely to develop neurological complications even though 
TABLE 5. Logistic regression analysis

\begin{tabular}{|c|c|c|c|c|c|c|}
\hline \multirow[b]{2}{*}{ Variable } & \multicolumn{3}{|c|}{ Univariate } & \multicolumn{3}{|c|}{ Multivariate } \\
\hline & OR & $95 \% \mathrm{Cl}$ & p Value & OR & $95 \% \mathrm{Cl}$ & $\mathrm{p}$ Value \\
\hline \multicolumn{7}{|l|}{ Decline in mRS score at early FU } \\
\hline Awake surgery & 40.5 & 3.8119 to 430.2932 & 0.0021 & 40.5 & 3.8119 to 430.2932 & 0.002 \\
\hline Globus pallidus & 13.9091 & 1.5399 to 125.6341 & 0.0191 & - & - & NS \\
\hline IC involvement & 8.5556 & 1.6828 to 43.4967 & 0.0097 & - & - & NS \\
\hline Weakness & 7.2 & 1.4578 to 35.3176 & 0.0150 & - & - & NS \\
\hline \multicolumn{7}{|l|}{ Decline in $\mathrm{mRS}$ score at last FU } \\
\hline Awake surgery & 19.3333 & 2.4341 to 153.5577 & 0.0051 & 19.3333 & 2.4341 to 153.5577 & 0.005 \\
\hline Worsening mRS score at early FU & - & NS & & & & \\
\hline \multicolumn{7}{|l|}{ Decline in mRS score at early FU* } \\
\hline Globus pallidus & 13.9091 & 1.5399 to 125.6341 & 0.0191 & - & - & NS \\
\hline IC involvement & 8.5556 & 1.6828 to 43.4967 & 0.0097 & 8.5556 & 1.6828 to 43.4967 & 0.009 \\
\hline Weakness & 7.2 & 1.4578 to 35.3176 & 0.0150 & - & - & NS \\
\hline \multicolumn{7}{|l|}{ Decline in mRS score at last FU* } \\
\hline Globus pallidus & - & NS & - & - & - & NS \\
\hline IC involvement & - & NS & - & - & - & NS \\
\hline Weakness & - & NS & - & - & - & NS \\
\hline Worsening mRS score at early FU & - & NS & - & - & - & NS \\
\hline
\end{tabular}

NS $=$ not significant.

Boldface type indicates statistical significance.

*Elimination of awake surgery as a confounder.

they tended to present with on average a larger lesion size. Surgical outcomes did not differ among the different surgical approaches.

Cerebral CM is the second most common vascular malformation identified on brain MRI ${ }^{8,9}$ The estimated prevalence of $\mathrm{CM}$ is 1 in 625 asymptomatic patients. CMs are often identified incidentally but can also present with new focal NDs, seizures, or nonspecific symptoms such as headaches. ${ }^{10}$ The management of cerebral CM remains controversial because the natural history of the lesion is not well understood. Previous natural history studies have estimated an annual symptomatic hemorrhage rate of $0.7 \%-6.5 \%$ per year and an asymptomatic hemorrhage rate of up to $13 \%$ based on retrospective studies. ${ }^{11,12}$ Risk factors such as a hemorrhagic presentation, deep anatomical location, and female sex increase the risk of developing a new symptomatic hemorrhage. ${ }^{13,14}$ Patients presenting with a previously symptomatic hemorrhage had an annual reported symptomatic rehemorrhage rate between $4.5 \%$ and $22.9 \%$ per year. ${ }^{15,16}$ Patients with CMs located in deep locations such as the basal ganglia, thalamus, or brainstem were also significantly more likely to present with neurological disability from a repeat hemorrhage than were patients with superficial lesions (4.1\%/yr vs 0\%/yr). ${ }^{4}$ Authors of a recent meta-analysis of predominantly prospective cohorts have estimated an $18.4 \%$ 5-year risk of symptomatic hemorrhage in nonbrainstem CMs presenting with hemorrhage or NDs as compared to a $30.8 \%$ risk in patients with brainstem CMs presenting with hemorrhage or NDs. The estimated 5-year risk in patients with brainstem CMs presenting without hemorrhage or focal deficits was $8.0 \%$ versus $3.8 \%$ in those with nonbrainstem lesions. ${ }^{17}$ Furthering our understanding of the natural history versus the surgical risks of BGCMs is important in guiding management.

The general attitude within the neurosurgical community toward the surgical management of eloquent and deep CMs such as BGCMs is conservative; as a result, the published literature is limited to case reports and very small series whose surgical outcomes are unclear. Gross et al. performed a systematic review of basal ganglia and thalamic CMs in 2008 and found that across surgical series and case reports with 103 patients, the overall gross-total resection rate was $89 \%$ with a $10 \%$ risk of long-term surgical morbidity and $1.9 \%$ risk of death. ${ }^{1}$ These results mirror our own, although there were no cases of surgical death in our series. The total number of BGCMs in that systematic review was 22, 14 of which were located in the caudate nucleus and 8 of which were in the lentiform nucleus. ${ }^{1}$ Since 2008 another 9 studies detailing 55 cases have been contributed to the literature (Supplementary Table 1). Our systematic review of the literature revealed a total of 90 BGCMs, and combined with the current series of 44 cases, the gross-total resection rate is $95.3 \%$ (102 of 107) with an $8.9 \%$ (12 of 134) risk of long-term neurological decline and 0 cases of surgically related deaths (Supplementary Tables $1-4)$. Further, $56.5 \%$ of patients in the systematic review were male with lesions involving the following areas of the basal ganglia: $35.3 \%$ caudate nucleus, $55.8 \%$ putamen, and $45.5 \%$ globus pallidus. An acute hemorrhagic presentation on radiographic imaging was reported in $73.9 \%$ of BGCMs in the literature. The most common clinical presentation was motor deficits $(44.1 \%)$, followed by seizure $(24.4 \%)$ and sensory deficit (14.2\%). The majority of surgeries involved the transsylvian approach $(66.7 \%)$, fol- 

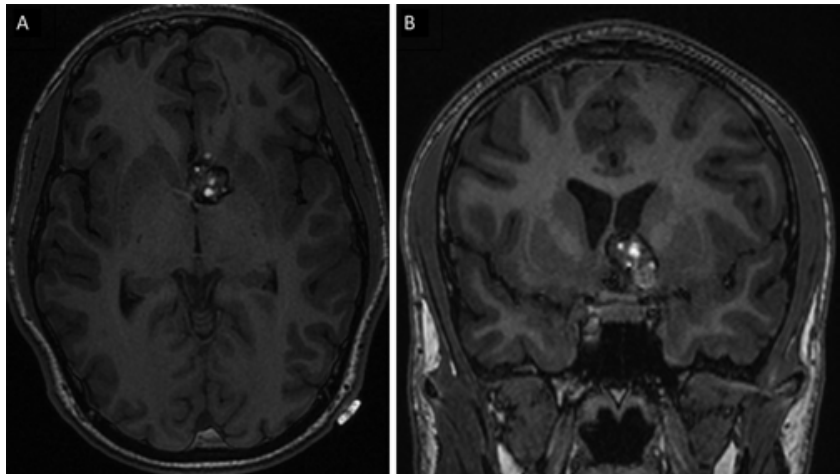

FIG. 3. Case 1. MRI demonstrated a $3 \times 2-\mathrm{cm}$ hemorrhagic mass within the left basal ganglia and involving the anterior commissure and anterior hypothalamus (A). The lesion presented to the third ventricle and floor of the lateral ventricle and was therefore a good candidate for an interhemispheric transcallosal transventricular approach (B).

lowed by the transcortical (23\%), transcallosal (5.7\%), and subfrontal (4.6\%) approaches. Supplementary Tables 2-4 list each study's presenting signs and symptoms, surgical approaches, and outcomes, respectively.

The present series contributes nearly half the total number of BGCMs previously published in the literature. Altogether, our experience and those of others demonstrate that favorable results can be achieved with resection and that an aggressive approach can be taken for lesions presenting with hemorrhage and/or NDs given the poor natural history of such lesions.

Patient selection and surgical approach are the most important factors in reducing surgical morbidity. Lesions involving the caudate nucleus, although larger at presentation, were less likely to be associated with perioperative deficits, whereas lesions involving the internal capsule were more at risk of causing transient surgical morbidity. The most logical explanation for these findings is the proximity to the corticospinal tracts; however, these cases can still be safely surgically treated without an increased risk of permanent morbidity depending on the adjunct studies used such as tractography. Awake craniotomy was found to be predictive of developing both transient and permanent morbidity. We hypothesize that because awake craniotomy is typically performed for higher-risk lesions involving or adjacent to the internal capsule, patients who had undergone this procedure were therefore more likely to suffer permanent morbidity given that these lesions had a higher baseline risk; however, increased surgeon confidence influenced by performing an awake craniotomy cannot be ruled out as a potential contributor.

Although outcomes did not differ among the different surgical approaches, approach selection is critical. We perform all BGCM surgeries with MEP/SSEP neuromonitoring and frameless stereotactic navigation. Diffusion tensor imaging is frequently performed to evaluate the anatomical location of eloquent tissue, and its avoidance is key when determining the optimal surgical approach. We prefer to avoid transcortical dissection if possible; therefore, our strategy heavily favored transsylvian (lesions involving the lentiform nucleus) and interhemispheric (lesions involving the caudate nucleus) trajectories, although
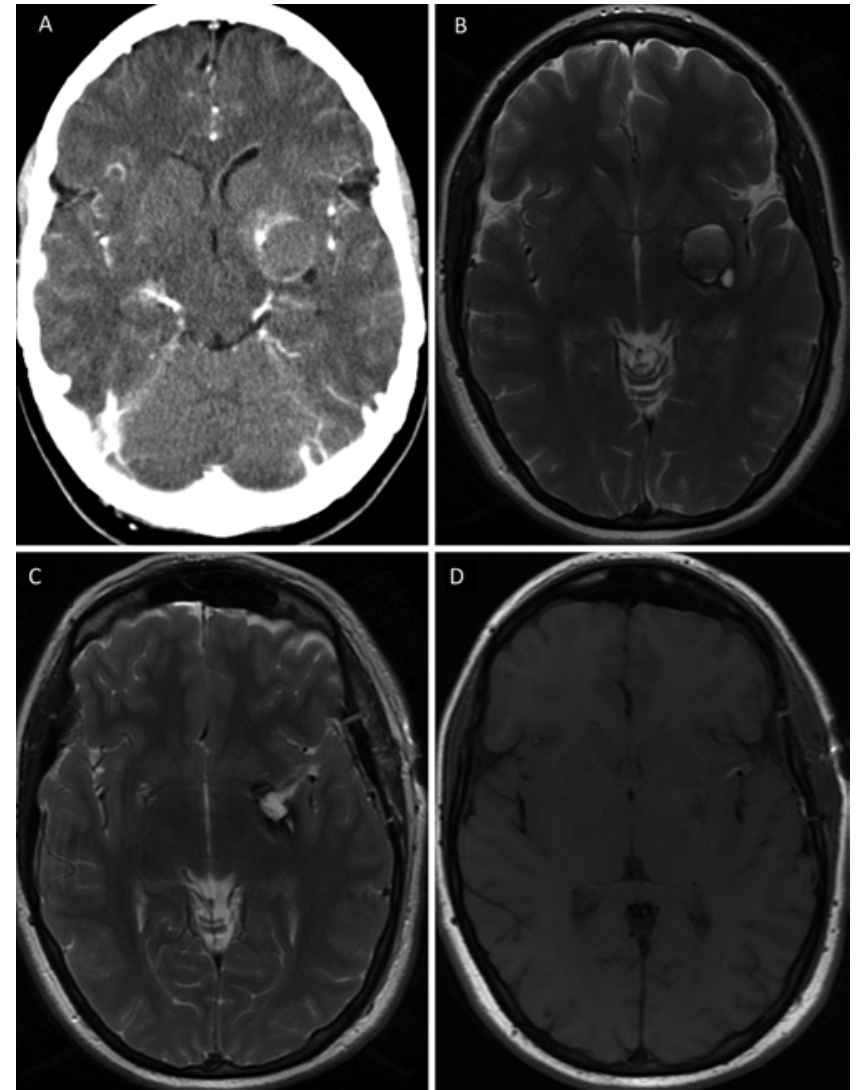

FIG. 4. Case 2. CTA demonstrated a 3-cm spherical hemorrhagic mass with a "spot sign" and fluid-fluid level concerning for a partially thrombosed aneurysm (A). MRI revealed a CM with acute and subacute hemorrhage within the left lentiform nucleus (B). Postoperative MRI demonstrated gross-total resection achieved via a transsylvian transinsular approach (C and D).

the transcortical and supracarotid infrafrontal approaches were performed in select cases. We have also previously described our experience using a carbon dioxide laser to reduce thermal damage and brain manipulation compared to those with traditional bipolar electrocautery. ${ }^{7}$ Alternative strategies for treating BGCMs include radiosurgery, but there is no proven benefit of this treatment modality on $\mathrm{CMs} .{ }^{18}$ At this time, we continue to advocate surgical management for BGCMs in symptomatic patients presenting with hemorrhage.

A limitation of this study is that it analyzes a singlecenter experience with a small cohort of patients managed over 3 decades. Although we performed a systematic review, the cases published in the literature are likely to be highly selective, and often only positive results are reported. Therefore, the nature of this paper lends itself to possible selection and assessor bias. Although patients presenting with hemorrhagic CMs have the potential for recovery, they are also prone to suffer repeat hemorrhages. The lack of a control group in our series precludes accurate assessment of the utility of resection. It is also difficult to generalize our experience in operating on a rare disease pathology to the broader community in treating these challenging lesions, especially since we have access 
to multiple intraoperative resources and extensive prior surgical knowledge, which may not be available to all neurosurgeons. This highlights the potential disparity between high-volume centers and other institutions. ${ }^{19}$ Nonetheless, the present study supports the safety of the surgical management of BGCMs in experienced hands. Future prospective multicenter registries are needed to validate our findings.

\section{Conclusions}

Surgery is a safe and effective treatment modality for managing BGCMs, with an estimated permanent morbidity rate of around $10 \%$. Patients with lesions involving the internal capsule are at higher risk for developing perioperative complications, whereas lesions involving the caudate are generally safer to resect. Surgical outcomes did not differ among the different surgical approaches; however, minimizing brain transgression and retraction should always be considered.

\section{Acknowledgments}

This study was supported in part by Bernard and Ronni Lacroute and the William Randolph Hearst Foundation (G.K.S.).

\section{References}

1. Gross BA, Batjer HH, Awad IA, Bendok BR. Cavernous malformations of the basal ganglia and thalamus. Neurosurgery. 2009;65(1):7-19.

2. Lorenzana L, Cabezudo JM, Porras LF, et al. Focal dystonia secondary to cavernous angioma of the basal ganglia: case report and review of the literature. Neurosurgery. 1992;31(6): $1108-1112$.

3. Thobois S, Jouanneau E, Bouvard M, Sindou M. Obsessivecompulsive disorder after unilateral caudate nucleus bleeding. Acta Neurochir (Wien). 2004;146(9):1027-1031.

4. Porter PJ, Willinsky RA, Harper W, Wallace MC. Cerebral cavernous malformations: natural history and prognosis after clinical deterioration with or without hemorrhage. J Neurosurg. 1997;87(2):190-197.

5. Chang EF, Gabriel RA, Potts MB, et al. Supratentorial cavernous malformations in eloquent and deep locations: surgical approaches and outcomes. Clinical article. J Neurosurg. 2011;114(3):814-827.

6. Abla AA, Spetzler RF, Albuquerque FC. Trans-striatocapsular contralateral interhemispheric resection of anterior inferior basal ganglia cavernous malformation. World Neurosurg. 2013;80(6):e397-e399.

7. Choudhri O, Karamchandani J, Gooderham P, Steinberg GK. Flexible omnidirectional carbon dioxide laser as an effective tool for resection of brainstem, supratentorial, and intramedullary cavernous malformations. Neurosurgery. 2014; 10(suppl 1):34-45.

8. Vernooij MW, Ikram MA, Tanghe HL, et al. Incidental findings on brain MRI in the general population. $N$ Engl J Med. 2007;357(18):1821-1828.

9. Morris Z, Whiteley WN, Longstreth WT Jr, et al. Incidental findings on brain magnetic resonance imaging: systematic review and meta-analysis. BMJ. 2009;339:b3016.

10. Al-Shahi Salman R, Berg MJ, Morrison L, Awad IA. Hemorrhage from cavernous malformations of the brain: definition and reporting standards. Stroke. 2008;39(12):3222-3230.
11. Kim DS, Park YG, Choi JU, et al. An analysis of the natural history of cavernous malformations. Surg Neurol. 1997;48(1): 9-18.

12. Zabramski JM, Wascher TM, Spetzler RF, et al. The natural history of familial cavernous malformations: results of an ongoing study. J Neurosurg. 1994;80(3):422-432.

13. Washington CW, McCoy KE, Zipfel GJ. Update on the natural history of cavernous malformations and factors predicting aggressive clinical presentation. Neurosurg Focus. 2010; 29(3):E7.

14. Al-Shahi Salman R, Hall JM, Horne MA, et al. Untreated clinical course of cerebral cavernous malformations: a prospective, population-based cohort study. Lancet Neurol. 2012; 11(3):217-224.

15. Aiba T, Tanaka R, Koike T, et al. Natural history of intracranial cavernous malformations. J Neurosurg. 1995;83(1): $56-59$.

16. Moriarity JL, Wetzel M, Clatterbuck RE, et al. The natural history of cavernous malformations: a prospective study of 68 patients. Neurosurgery. 1999;44(6):1166-1173.

17. Horne MA, Flemming KD, Su IC, et al. Clinical course of untreated cerebral cavernous malformations: a meta-analysis of individual patient data. Lancet Neurol. 2016;15(2):166-173.

18. Pollock BE, Garces YI, Stafford SL, et al. Stereotactic radiosurgery for cavernous malformations. J Neurosurg. 2000; 93(6):987-991.

19. Davies JM, Lawton MT. Improved outcomes for patients with cerebrovascular malformations at high-volume centers: the impact of surgeon and hospital volume in the United States, 2000-2009. J Neurosurg. 2017;127(1):69-80.

\section{Disclosures}

Dr. Steinberg is a consultant for Qool Therapeutics, Peter Lazic US, NeuroSave, SanBio, Audaxion Therapeutics, Zeiss, and Surgical Theater.

\section{Author Contributions}

Conception and design: Li. Acquisition of data: Li, Khahera. Analysis and interpretation of data: Steinberg, Li, Khahera, Kim, Han. Drafting the article: Steinberg, Li, Khahera, Mandel. Critically revising the article: Steinberg, Li, Khahera, Kim, Han. Reviewed submitted version of manuscript: all authors. Approved the final version of the manuscript on behalf of all authors: Steinberg. Statistical analysis: Kim, Han. Administrative/ technical/material support: Steinberg. Study supervision: Steinberg.

\section{Supplemental Information \\ Videos}

Video 1. https://vimeo.com/451122955.

Video 2. https://vimeo.com/451124163.

\section{Online-Only Content}

Supplemental material is available with the online version of the article.

Supplementary Tables 1-4. https://thejns.org/doi/suppl/ 10.3171/2020.7.JNS2098.

\section{Correspondence}

Gary K. Steinberg: Stanford University School of Medicine, Stanford, CA. gsteinberg@stanford.edu. 